\title{
SMOOTH BANACH ALGEBRAS ${ }^{1}$
}

\author{
I. N. $\operatorname{SPATZ}^{2}$
}

Introduction. The purpose of this note is to prove the theorem that follows. This theorem generalizes results of L. Ingelstam [2] and M. F. Smiley [4] for Hilbert algebras. It also provides a simpler proof of Corollary which was obtained by F. F. Bonsall and J. Duncan [1]. (This result was obtained before I was aware of the work of Bonsall and Duncan.)

Let $G(x, y)\left(G_{+}(x, y), G_{-}(x, y)\right)$ denote the limit as $t$ approaches zero (from the right, from the left) of $(\|x+t y\|-\|x\|) / t$. For any of the properties of $G$ used in the proof see Koethe [3].

THEOREM. Let $X$ be a real Banach algebra with identity $e$. $\|e\|=1$. If $G(e, s)$ exists for each singular element $s$ of $X$, then $X$ is a division algebra.

Proof. If $s \in X$ is singular, then $G(e, s)=0$ since $\|e+t s\| \geqq 1$ implies $G_{+} \geqq 0$ and $G_{-} \leqq 0$.

It follows immediately that $e+s$ is invertible for $G(e, e+s)=G(e, e)$ $+G(e, s)=1$.

Suppose $u \in X$ is invertible and $\|u\|=1$. Then $\|u+t s\| \leqq\left\|e+t u^{-1} s\right\|$. It follows immediately from the above inequality (and the fact that $u^{-1} s$ is singular if $s$ is) that $G_{+}(u, s) \leqq G_{+}\left(e, u^{-1} s\right)=0$ and $G_{-}(u, s)$ $\geqq G_{-}\left(e, u^{-1} s\right)=0$. But, in general, $G_{+} \geqq G_{-}$. Thus $G(u, s)=0$.

If $s$ is singular, then $u_{\alpha}=(e+\alpha s) /\|e+\alpha s\|$ ( $\alpha$ a real number) is an invertible element of norm one. Hence

$$
1=G\left(u_{\alpha}, u_{\alpha}\right)=G\left(u_{\alpha}, e\right) /\|e+\alpha s\| .
$$

Since $G\left(u_{\alpha}, e\right) \leqq\left\|u_{\alpha}\right\|\|e\|=1$,

$$
1 \geqq\|e+\alpha s\| \geqq|\alpha|\|s\|-1 .
$$

Evidently, this implies $s=0$.

Corollary. If $X$ is a smooth Banach algebra over the reals with identity $e$ of norm one, then $X$ is the reals, complexes or the real quaternions.

Presented to the Society, February 25, 1967 under the title Geometry of banach algebras; received by the editors February 12, 1968.

1 Taken from a dissertation submitted to the Faculty of the Polytechnic Institute of Brooklyn in partial fulfillment of the requirements for the degree of Doctor of Philosophy (Mathematics), 1967.

2 The author held a National Science Foundation Traineeship during the time this research was done. 


\section{REFERENCES}

1. F. F. Bonsall and J. Duncan, Dually irreducible representations of Banach algebras, Yale Univ., New Haven, Conn., 1967, p. 18.

2. L. Ingelstam, Hilbert algebras with identity, Bull. Amer. Math. Soc. 69 (1963), 191-194.

3. G. Koethe, Topologische Lineare Raume, Springer-Verlag, Berlin, 1960, pp. 351-352.

4. M. F. Smiley, Real Hilbert algebras with identity, Proc. Amer. Math. Soc. 16 (1965), 440-441.

Brookl yN College 